\section{Pollination and Seed Production in Viola}

\author{
Daiichiro Miyajima ${ }^{1}$ \\ Faculty of Agriculture, Miyazaki University, Miyazaki, Miyazaki, \\ Japan 889-2192
}

\author{
Additional index words. pollen characteristics, flower longevity, stigma receptivity, Viola \\ $\times$ cornuta
}

\begin{abstract}
The seed producing system in viola (Viola $\times$ cornuta) was investigated to improve seed yield and to save labor. In a flower five anthers sequentially dehisced; pollen grains were continuously supplied to the anterior petal, which played a significant role in pollination, throughout the flowering period. Evidence from pollen and ovule number suggests that the species is facultative autogamy. Each flower opened more than $\mathbf{1 0}$ days was independent of the success in fertilization and kept seed producing ability during the flower longevity period. Pollen grains also maintained viability during the flower longevity period. Pollinators were indispensable for pollination of viola, but pollination in viola was done by a different mechanism from the typical insect-mediated pollination that sticky pollen grains adhere to the exposed stigmas. Pollen grains, accumulated around the entrance of the stigmatic cavity, entered into the cavity by the movement of pollinators. Although the visitation of pollinators was occasional, solitary bees primarily contributed to the pollination of viola. On the other hand, germination of pollen grains on the stigmatic surface was under $50 \%$. Seed set was much lower than the germination percentage of pollen grains. A viola flower had the ability for additional pollinations and fertilization for some days after the fertilization success in some ovules in the flower. This characteristic suggested that repeated pollination is effective to increase the number of mature seeds in a capsule.
\end{abstract}

Viola (Viola $\times$ cornuta) is a popular bedding plant. The need for viola seeds is great. Because several flowers newly open every day in each viola plant and mature seeds are easily dispersed by autonomous capsule cracking, seed production of viola requires much labor which makes the viola seeds high-priced. In plants with multiovulate flowers, increase in the number of mature seeds in a fruit improves seed yield and promotes labor cost reduction. Maximizing the number of mature seeds in each fruit is one of the critical factors for commercial viola seed production. Each viola flower stays alive for a long period. Since a long flowering period is a burden for plants, plants usually have a system to make their flowers die by endogenous ethylene following fertilization success (Clark et al., 1997; Halevy, 1995; Hilioti et al., 2000; Hoekstra and van Roekel, 1986; Ketsa and Rugkong, 2000; Larsen et al., 1995; Piskornik, 1986; Woodson et al., 1992). On the other hand, long flower life in multiovulate flowers may produce a greater number of mature seeds. It is commonly recognized that the ovary start to develop into the fruit following fertilization success. In viola, the occasional pollinator visitations and the structure of stigmas contribute to the stigmas receiving few pollen grains in a short period. If fertilization in a small number of ovules caused by a pollination with a small number of pollen grains blocks additional fertilization, the unfertilized ovules will abort. Therefore, the possibility of fertilization success after the first fertilization that triggers the development of the ovary into fruit is a theme to be clarified. In this experiment, I tried to uncover the seed

Received for publication 10 Oct. 2005. Accepted for publication 12 Jan. 2006.

${ }^{1}$ To whom reprint requests should be addressed; e-maila01104u@cc.miyazaki-u.ac.jp. production system in viola. Concretely, the themes are 1) the flowering characteristics from buds to full opening of petals and sequence in pollen production and supply, 2) the structure of the stigma and the characteristics of pollen, 3 ) flower longevity and seed producing ability in the flowering period, 4) kinds and behavior of pollinators, 5) seed production under some different breeding conditions.

\section{Materials and Methods}

This study was conducted at an unheated greenhouse in the Faculty of Agriculture, Miyazaki University in Miyazaki Prefecture, Japan from 2002 to 2005 . Temperatures during experiments were shown in Fig. 1. Seeds of 'Velour Cream Splash' (VCS; M\&B Flora Co., Ltd, Yamanashi, Japan) and 'Princess Blue'(PB; Sakata Seed Co., Yokohama, Japan) were sown in $20 \mathrm{~cm}$ flower pots made of clay filled with a commercially prepared peat-based soil mixture (Sotan growing medium; Green Farm Co., LTD, Fukuoka, Japan) on 5 Sept. 2002, 8 Sept. 2003, and 21 Sept. 2004. A $14 \mathrm{~N}-2.2 \mathrm{P}-5.8 \mathrm{~K}$ liquid fertilizer was applied weekly $\left(5 \mathrm{~g} \cdot \mathrm{L}^{-1}\right)$ after germination. Plants at two to three true leaf stage were transplanted into polyethylene pots $12 \mathrm{~cm}$ in diameter filled with the medium above described. When the plant canopy diameter became about $12 \mathrm{~cm}$, plants were transplanted into $20 \mathrm{~cm}$ flower pots made of clay filled with the medium. A $14 \mathrm{~N}-2.2 \mathrm{P}-5.8 \mathrm{~K}$ liquid fertilizer was applied weekly. Hand-pollination was done by the following procedures. In cases of pollination with a mass of pollen grains, pollen grains were placed on a pair of tweezers with a sharp edge. The pollen grains on the tweezers were pushed against the mucilage spreading inside the stigmatic cavity. In cases of pollination with a small fixed number of pollen grains, pollen grains were counted and were placed on the apex of a 0.8 -mm flat-blade screwdriver under a binocular microscope (KarlZeiss, Stemi DV4). Pollen grains on the screwdriver were placed on the stigmatic mucilage. Petals and anthers were removed after hand-pollination to prevent additional pollination by insects.

Number of days to full opening of petals and sequential anther dehiscence. In February and March 2003, the dates when a flower began to open and when the flower fully opened were recorded for 30 flowers in each cultivar. Angles made by the posterior and the anterior petals of flowers at different days from the beginning of opening of petals were measured and the anther dehiscence of each anther of the flower was judged.

Structure of the stigma and number of ovules in a flower. In January 2003, the whole area of the dome structure at the apex of the pistil of cut flowers was dusted with pollen grains from flowers that had fully opened. The cut flowers were set at $20^{\circ} \mathrm{C}$ following the removal of petals and anthers. After $6 \mathrm{~h}$, the dome structures were longitudinally cut by a surgical knife and the germination of pollen grains was observed under a binocular microscope following staining with $1 \%$ acetocarmine. The number of ovules of 20 flowers in each cultivar was counted under a binocular microscope.

Number of pollen grains and pollen viability duringflower longevity. In February and March 2004 , the number of pollen grains of a flower was counted for 20 flowers in each cultivar by the following procedures. Anthers just before dehiscence were excised from flowers in which posterior and anterior petals made an angle of 90 degrees (hereafter, the angle made by the posterior and the anterior petals is called 'petal angle'). An excised anther was placed in a drop of glycerin on a slide glass. Pollen grains were swept from the anther by needles and the number of pollen grains was counted under a binocular microscopes. The number of pollen grains in an anther $\times 5$ was considered to be that of a flower. Germination of pollen on the stigma was assessed by the following procedures. The dome structures at the apexes of pistils of flowers that had fully opened were longitudinally cut by a surgical knife under a binocular microscope. The layer of mucilage inside the stigmatic cavity was pushed on to glass slides. Pollen grains that accumulated between the stigma and the anterior petals of the flowers that had fully opened were collected and were scattered on the mucilage daubed on the glass slides. The glass slides were placed in petri dishes together with wet cotton at 20 ${ }^{\circ} \mathrm{C}$ for $3 \mathrm{~h}$. The number of germinating and nongerminating pollen grains was counted under a light microscope. A pollen grain was considered to have germinated when the length of the pollen tube was equal to or longer than the diameter of the grain. The germination percentage of pollen grains was calculated. The duration of pollen viability was determined by the following procedures. Flowers that had fully opened in plants covered by a mosquito net were tagged. Pollen grains that remained in the inferior anthers were collected 6 to $10 \mathrm{~d}$ after full opening of the flower. Flowers with 
the petal angle 60 to $90^{\circ}$ were pollinated with the pollen grains. Developing capsules were sampled about 20 DAP and the number of seeds were counted. Differences in the pollen viability among the days from anther dehiscence to pollination and between cultivars were tested by the analysis of variance.

Number of seeds in a capsule formed by a flower pollinated with a different number of pollen grains. In February and March 2004, flowers with petal angles 60 to $90^{\circ}$ were pollinated with $10,20,30$, and 40 pollen grains picked from the superior anthers excised from flowers with the petal angles 30 to $45^{\circ}$. Capsules were harvested about $20 \mathrm{DAP}$ and the number of seeds in a capsule was counted. Differences in the number of seeds in a capsule and fruit set among the number of pollen grains used for pollination and between cultivars were analyzed by the analysis of variance. Percentage of fruit set were arcsine transformed before analysis.

Flower longevity and female function. Plants covered by a mosquito net were used. In the following experiments, pollinations were performed with a mass of pollen grains obtained from other flowers that had fully opened except for the pollination with 30 grains. All flowers pollinated were harvested about $20 \mathrm{~d}$ after pollination and the number of seeds in a capsule was counted. In February and March 2003, more than 20 flowers in each cultivar with petal angles 15 to $45^{\circ}$ were emasculated or pollinated. In both treatments, the days from full opening to the beginning of withering of the flower were recorded and the status of the stigma during this period was observed. For pollinated flowers, seed set was confirmed by the growth of capsules more than 20 DAP. Flowers with petal angles 15 to $45^{\circ}$ were emasculated and were pollinated 0 to $10 \mathrm{~d}$ after the full opening of the flower. Differences in the number of seeds in a capsule between the cultivars and among the days from the full opening of the flower to pollination were analyzed by the analysis of variance. To estimate the time from pollination to fertilization, 10 flowers of VCS that had fully opened were pollinated. Styles of these flowers were removed $24 \mathrm{~h}$ after pollination. In February and March 2005, flowers that had fully opened were pollinated with 30 pollen grains. Petals and anthers of these pollinated flowers were removed. These flowers were pollinated again with 30 or a mass of pollen grains 1 to $8 \mathrm{~d}$ after the first pollination. Differences in the number of seeds in a capsule among the days between the first and the second pollination including single pollination were analyzed by the analysis of variance. Dunnett's test was used to compare the single and double pollination.

Seed production under some different conditions. In February, March, and April 2003, plants were treated as follows: 1) open pollination; 2) covering with a mosquito net (1 $\mathrm{mm}$ mesh); 3) petals of flowers with the petal angle 0 to $15^{\circ}$ were removed, 4) emasculation of flowers with petal angle 15 to $45^{\circ} ; 5$ ) flowers that had fully opened were hand-selfed. Five plants were used for each treatment in each cultivar. Capsules were sampled about $20 \mathrm{~d}$ after opening and the number of seeds was counted. Differences in the number of seeds in a capsule among treatments were analyzed by the analysis of variance. Scheffe's test was used to compare the effects of treatments. Pollinators were observed throughout the total experiments from 0800 to $1200 \mathrm{HR}$ in $4 \mathrm{~d}$ per week and from 1300 to 1700 HR in a few days per week. Due to the rarity of pollinator visitation, frequency of visits was not measured.

\section{Results}

The number of days to full opening of petals and sequential anther dehiscence. Number of days from the beginning of opening to full opening of petals were $3.1 \pm 0.8$ (average \pm $\mathrm{SD})$ and $4.2 \pm 1.0$ for VCS and PB, respec- tively. In a flower, five anthers sequentially dehisced during the period from the beginning of opening to a few days after opening of the flower. Anther dehiscence began with the superior anther, followed by that of the two lateral anthers, and terminated with the two inferior anthers. In VCS, the superior anther was already dehisced when the petal angle became $90^{\circ}$ (Fig. 2). In both cultivars, superior and lateral anthers had already dehisced when the flower fully opened. The inferior anthers dehisced from the next day of full opening of the flower and terminated the dehiscence 4 and $3 \mathrm{~d}$ after full opening of the flower for VCS and $\mathrm{PB}$, respectively.

Structure of the stigma and number of ovules in a flower. Pollen grains germinated only on the layer of mucilage spreading inside
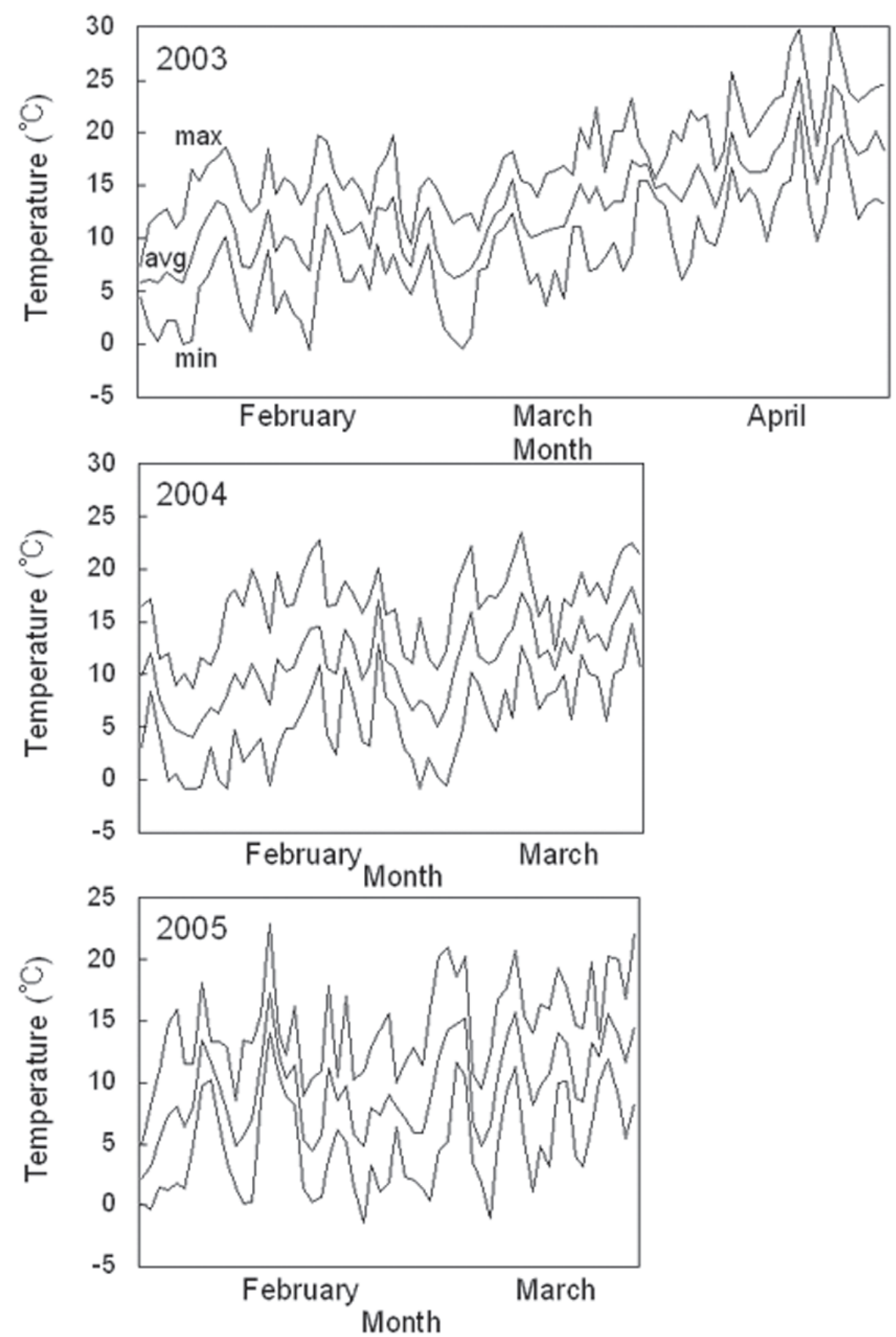

Fig. 1. Maximum, average, and minimum temperatures for each day during experiments. 

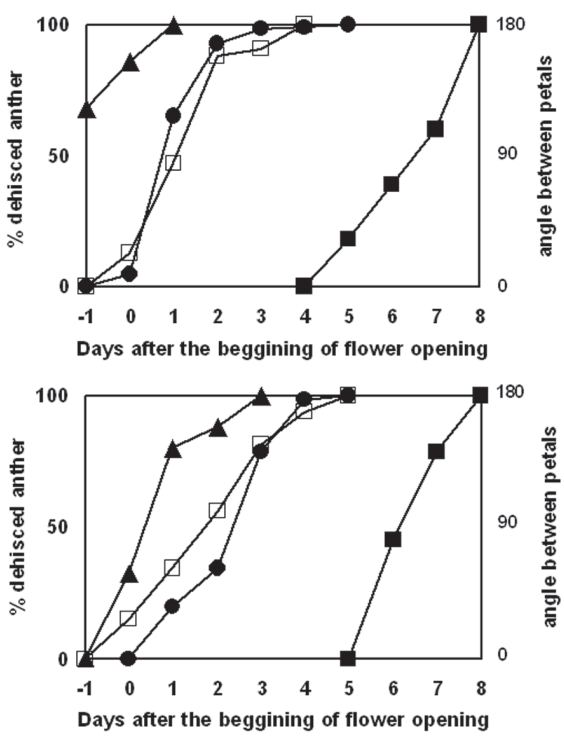

Fig. 2. Daily petal opening and anther dehiscence in the flowers of viola VCS (upper) and PB (lower). Day 0 denotes the day when the flower began to open. The empty square denotes the angle between the posterior and the anterior petals. The solid shapes are the percentages of dehisced anthers: $\boldsymbol{\Delta}$ superior anthers, $\bullet$ lateral

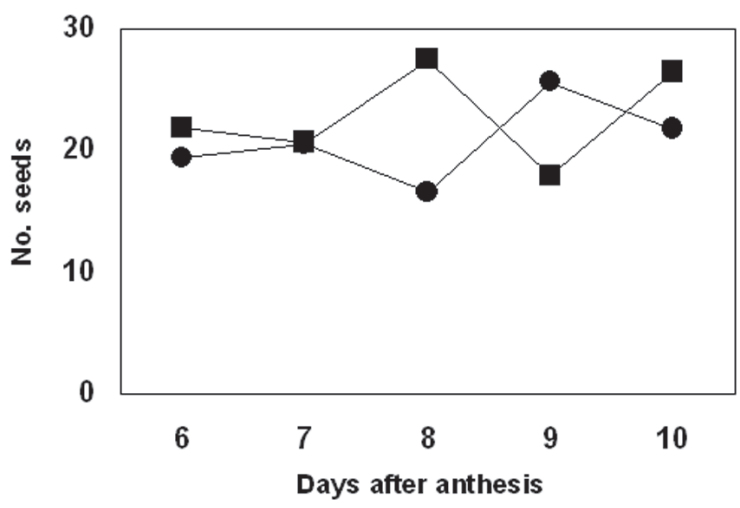
a flower pollinated with pollen grains in the inferior anthers at different days after full opening of the flower in two cultivars of viola. VCS, PB. anthers, $\mathbf{\square}$ inferior anthers.

Fig. 3. Number of seeds in a capsule formed by

the stigmatic cavity. Pollen grains adhered to the entrance of the stigmatic cavity to some extent, but no pollen grains germinated there. A flower of VCS and of PB had $65.9 \pm 3.1$ and $67.7 \pm 9.7$ ovules, respectively.

Number of pollen grains and pollen viability during flower longevity. Viola pollen grains have a nonsticky nature; these grains did not stick to each other and easily dropped from the dehisced anthers. A flower of VCS and of PB had $7020 \pm 676$ and $4810 \pm 670$ pollen grains, respectively. Germination percentages of pollen grains on the stigmatic mucilage were $46 \%$ and $37 \%$ for VCS and PB, respectively. Pollen grains on the inferior anthers of flowers $10 \mathrm{~d}$ after full opening showed seed producing ability equal to those obtained from flowers 6 d after full opening (Fig. 3).

Number of seeds in a capsule formed by a flower pollinated with different number of pollen grains. Pollinations with greater number of pollen grains resulted in higher fruit set and a greater number of seeds in a capsule (Table 1). In both cultivars, more than $70 \%$ of fruit set was achieved by pollinations with 30 pollen grains and above. Number of seeds in a capsule linearly regressed to the number of pollen grains used for pollination. The maximum number of seeds in a capsule was 20 ; that was achieved by pollination with 30 pollen grains.

Flower longevity and female function. The emasculated and pollinated flowers of VCS began to wither 10.9 and $10.7 \mathrm{~d}$, respectively, after full opening of the flower. These flowers of PB began to wither 10.0 and $10.9 \mathrm{~d}$, respectively, after full opening of the flower. There were no significant differences in these days between cultivars, and between emasculated and pollinated flowers. There were little changes in the appearance of the stigma during flower longevity. In both cultivars, pollination at full opening of the flower with a mass of pollen grains resulted in about 30 seeds in a capsule (Fig. 4). In $\mathrm{PB}$, the number of seeds in a capsule formed by a flower pollinated at different days after

Table 1. Seed set of flowers pollinated with different number of pollen grains in two cultivars of viola.

\begin{tabular}{|c|c|c|c|}
\hline \multirow{2}{*}{$\begin{array}{l}\text { No. pollen } \\
\text { grains }^{z}\end{array}$} & \multirow{2}{*}{$\begin{array}{c}\text { Fruit } \\
\text { set (\%) }\end{array}$} & \multicolumn{2}{|c|}{ No. mature seeds/flower } \\
\hline & & $\overline{\mathrm{Avg}}$ & Max \\
\hline \multicolumn{4}{|l|}{ Velour Cream Splash } \\
\hline 10 & 31.6 & 0.7 & 3 \\
\hline 20 & 48.3 & 1.3 & 6 \\
\hline 30 & 70.0 & 3.2 & 20 \\
\hline \multirow[t]{2}{*}{40} & 75.0 & 4.5 & 16 \\
\hline & $\mathrm{L}^{* * * *}$ & & \\
\hline \multicolumn{4}{|l|}{ Princess Blue } \\
\hline 10 & 38.0 & 0.9 & 8 \\
\hline 20 & 57.9 & 2.1 & 10 \\
\hline 30 & 75.3 & 3.5 & 20 \\
\hline \multirow[t]{2}{*}{40} & 75.5 & 5.6 & 20 \\
\hline & $\mathrm{L}^{* * *}$ & & \\
\hline \multicolumn{4}{|l|}{ Statistical significance } \\
\hline Between cultivar & NS & NS & \\
\hline Among number pollen grains & ** & $* * *$ & \\
\hline
\end{tabular}

${ }^{2}$ Number of pollen grains on the stigmatic surface.

${ }_{\mathrm{NS}, * * * * * *}^{*}$ Nonsignificant or significant at $P=0.01$ or 0.001 , respectively, by $\mathrm{F}$ test using arcsin-transformed data; $\mathrm{L}=$ linear opening linearly regressed to the number of days after opening of the flower. The number of seeds in a capsule decreased with the increase of days from the opening of the flower, but more than 10 seeds were formed by a flower pollinated $10 \mathrm{~d}$ after opening. For VCS, the number of seeds in a capsule did not decrease with the increase of the days from the opening of the flower. Flowers of VCS pollinated $10 \mathrm{~d}$ after opening formed 20 seeds. Flowers, styles of which were removed $24 \mathrm{~h}$ after pollination, set seeds. Single pollination with 30 pollen grains resulted in $76 \%$ and $72 \%$ fruit set for VCS and PB, respectively. In both cultivars, second pollination with 30 or a mass of pollen grains increased fruit set compared to single pollination (chi-square test for independence, $P<0.05$ ). Flowers pollinated with 8-d intervals between the twice pollinations showed comparable fruit set with flowers pollinated twice with shorter intervals (Fig. 5). Flowers of VCS and $\mathrm{PB}$ pollinated once with 30 pollen grains formed 3.5 and 3.3 seeds, respectively, in a capsule. In both cultivars, a single pollination with a mass of pollen grains resulted in about 30 seeds in a capsule. Additional pollination with 30 or a mass of pollen grains following the first pollination with 30 pollen grains increased the number of seeds in a capsule compared to capsules formed by the flowers pollinated once with 30 pollen grains (Fig. 6). Flowers of VCS pollinated again with a mass of pollen grains $8 \mathrm{~d}$ after the first pollination produced comparable number of seeds with those pollinated once with a mass of pollen grains at full opening of the flower. In contrast, flowers of PB pollinated again with a mass of pollen grains $8 \mathrm{~d}$ after the first pollination produced a smaller number of seeds than those pollinated once with a mass of pollen grains when they fully opened. In both cultivars, the maximum number of seeds in a capsule formed by a flower pollinated once with 30 pollen grains was 20 (Fig. 7). The maximum number of seeds in a capsule was greater with flowers pollinated again with 30 pollen grains $8 \mathrm{~d}$ after the first pollination than with flowers pollinated once. On the other hand, the maximum number of seeds in a capsule was smaller with flowers pollinated again with a mass of pollen grains after the first pollination than with those pollinated once with a mass of pollen grains. In a developing capsule formed from a flower pollinated twice, differences in the growth stages of seeds among fertilized ovules were clearly observed (Fig. 8).

Seed production under some different conditions. The visit of pollinators to viola was rare throughout the experiment. Three kinds of solitary bees foraging only for nectar were occasionally observed. No visitation of honeybees, which were frequently observed in the experimental field, to viola was observed. Solitary bees landed on anterior petals of viola flowers and collected nectar facing their heads to the entrance of the stigmatic cavity and inserting their proboscis between the stigma and the anterior petals where pollen grains accumulated. At that moment, pollen grains were moved around the entrance of the stigmatic cavity and entered into the stigmatic 
cavity. Pollen grains adhered to the mouthparts of solitary bees just after sucking the nectar. Solitary bees often wiped pollen grains on their mouthparts. A large number of pollen grains adhered to the entrance of the stigmatic cavity of flowers visited by solitary bees. Flowers emasculated or with petal removal did not set seed (Table 2). There were great differences in

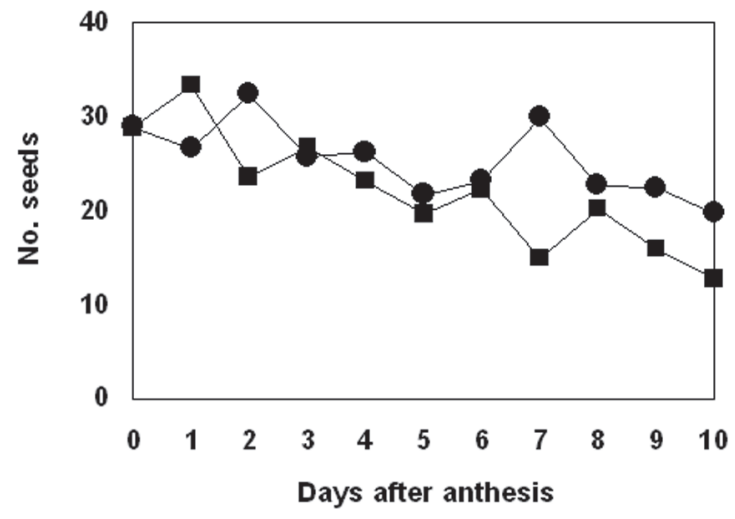

Fig. 4. Number of seeds in a capsule formed by a flower pollinated at different days after full opening in two cultivars of viola: VCS, $\boldsymbol{D B B}$.
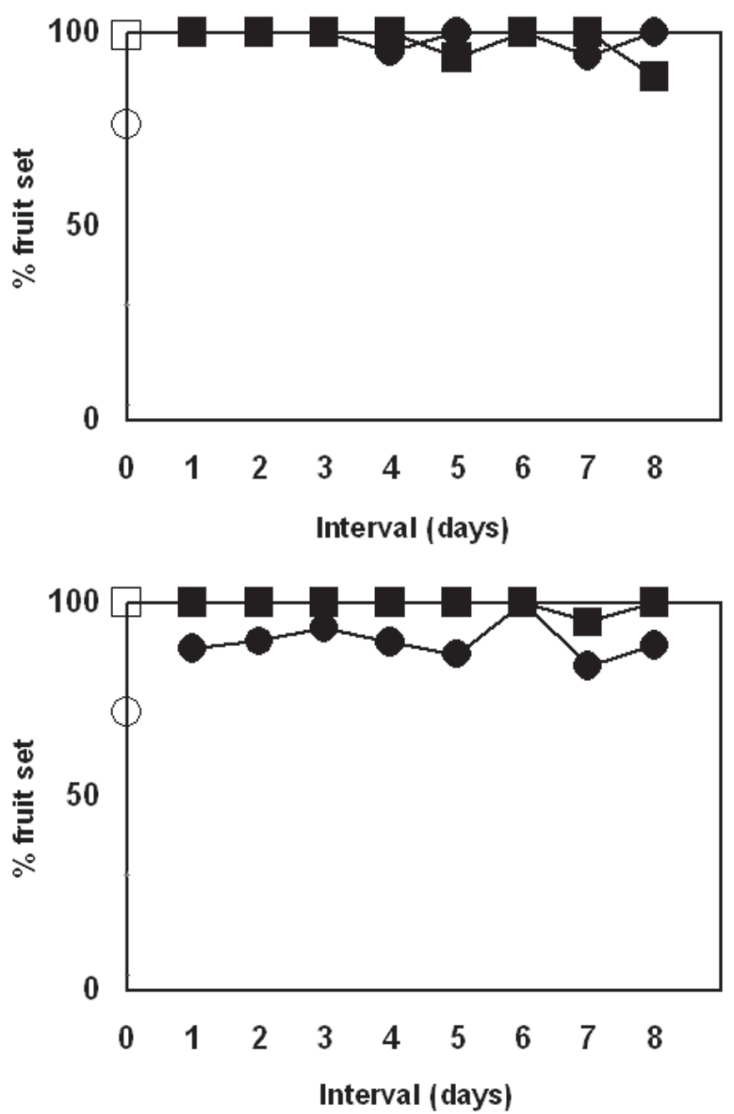

Fig. 5. Fruit set of flowers pollinated with 30 pollen grains at full opening of the flower and pollinated again with 30 or a mass of pollen grains in two cultivars of viola. (upper) VCS, (lower) PB. The $\mathrm{X}$-axis number denotes the intervals of days between the first and the second pollination: first pollination with 30 pollen grains and second pollination with a mass of grains, each of the twice pollinations with 30 pollen grains, $\square$ single pollination with a mass of pollen grains, O single pollination with 30 pollen grains. of all anthers in a flower after anthesis (Beattie, 1969a). Germination of pollen

seed production between flowers under open pollination and those covered by mosquito nets. In VCS, no seeds were produced under mosquito nets. In VCS, fruit set was higher with hand-pollinated flowers than with flowers under open pollination. In both cultivars, the number of seeds in a capsule was greater with flowers under open pollination than with hand-pollinated flowers.

\section{Discussion}

In viola, pollen grains were supplied to the anterior petals contacting with the stigma consecutively from the petal opening to a few days after full opening of the flower by a sequential anther dehiscence mechanism. Freitas and Sazima (2003) observed that dehiscence of anthers follows a superior-lateral-inferior sequence in Viola cerasifolia and Viola subdimidiata. In three wild species of grains on the stigma revealed that the stigmatic surface was the layer of mucilage spreading inside the stigmatic cavity. The mucilageous layer spreading inside the cavity appears not to be functional for pollination. I assume that the stigmatic structure is an adaptation to protect the stigmatic surface by preventing desiccation or adhesion of foreign objects during the flower longevity. The nonsticky nature of the pollen grains is an adaptation for pollination success in the cavity structure (Beattie, 1971). On the other hand, no honeybees visited viola and no solitary bees collected pollen grains. Pollen grains with a nonsticky nature may be hard to carry for pollinators or may not be suitaverage pollen viability of Viola cerasifolia was $72.5 \%$ (Freitas and Sazima, 2003). In my experiment, although germination percentage of pollen grains on the stigma mucilage was under $50 \%$, pollen grains kept viability for a long period. Pollen of Viola riviniana kept viability for $8 \mathrm{~d}$ (Beattie, 1969a). On the other hand, the possibility of adhesion of a mass of pollen grains on the stigmatic surface in a short due to the cavity structure and the nonsticky pollen grains. Repeated pollinations with each pollination having a small number of pollen grains adhere may actually happen. According to the results of pollination with a small number of pollen grains, the number able for feeding their larvae. The time is low in viola under open pollination of seeds in a capsule was only about $10 \%$ of that of pollen grains used for pollination. In spite of the short style of viola, actual seed set did not reflect the germination percentage of pollen grains on the stigma. The cause of the difference between the number of pollen grains on the stigma and the actual seed set is needed to be investigated further.

In viola, there were little differences in flower longevity between fertilized and unfertilized flowers. For plants, keeping the flower alive after the fertilization success wastes resource. In viola flowers, nectar is secreted deep in the concealed flower structure. This structure limits pollinators. Viola seems to decrease the risk for the failure in seed formation by increasing the flower longevity. Flowers with petal angles $15^{\circ}$ or at the beginning of petal opening set seeds following pollination, which indicates the maturation of ovules at full opening of the flower. Flowers kept alive after fertilization success without deterioration of the stigma. The results of the effects of second pollination after fertilization success confirmed that the remaining unfertilized ovules in a viola flower in which some ovules had been fertilized can be fertilized by the second pollination even if there is a interval of several days between the two pollination.

Thus, a viola flower has an extended reproduction period being independent of fertilization success. The flower longevity may also enhance pollen donation to other plants. In many plants, ethylene operates following pollination (Larsen et al., 1995). Flowers abscise, wilt or close reacting to ethylene (van Doorn, 2002). Ethylene is indispensable for ovary growth following pollination of some plants (Ketsa and Rugkong, 2000). Many flowers lose pollinator attractiveness by the change of color and form of petals following fertilization success (van Doorn and Stead, 1997). On the other hand, floral longevity change flexibly depending on the number of fertilized ovules in some plants (Ishii and Sakai, 2000). Viola flowers maintained pollinator attractiveness independent of pollination or fertilization success possibly by being free from ethylene production. In most plants, fruits develop after fertilization(Vivian-Smith etal., 2001). Further study is necessary to uncover how pollen tubes pass through the ovary that begins to develop into fruits in viola.

The pollen to ovule ratio indicated that the viola is facultative autogamy according to the criterion suggested by Cruden(1977). Beattie (1972b) reports the pollen flow among wild species of viola by pollinator's bodies. Freitas and Sazima (2003) observed bees collecting pollen in two wild species of viola. In my experiment, pollen grains were scarcely observed on pollinator's bodies visiting viola except for their proboscises after sucking the nectar. With the results that emasculated flowers hardly set seed, viola seeds were produced primarily by self-pollination performed by pollinators' movement. A mass of self pollen grains at the entrance of stigmatic cavity after pollinator's visitation also indicates the low frequency of cross-pollination. In Viola 

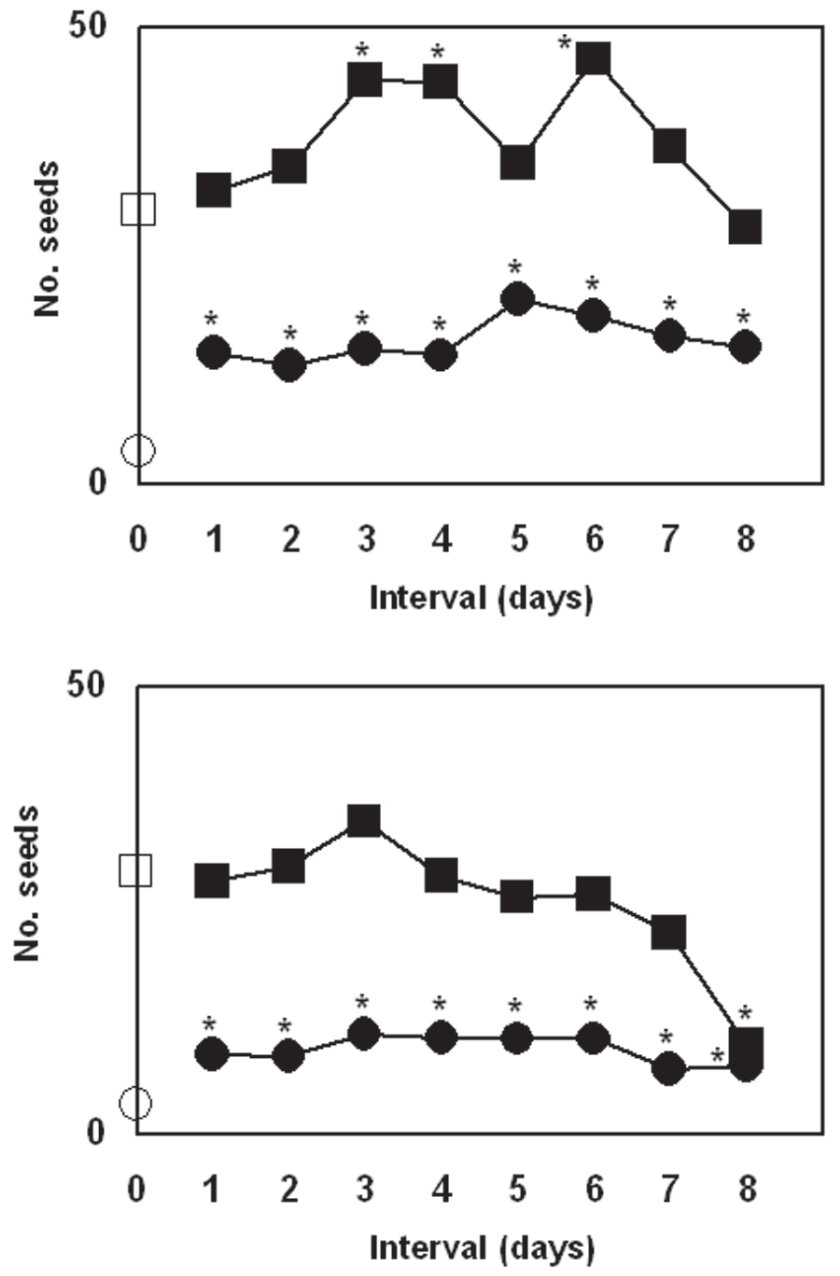

Fig. 6. Average number of seeds in a capsule formed by a flower pollinated with 30 pollen grains at full opening of the flower and pollinated again with 30 or a mass of pollen grains in two cultivars of viola. (upper) VCS, (lower) PB. The representation of shapes in graphs is the same as in Fig. 4. The asterisks on shapes denote that the number of seeds in a capsule is significantly different between the capsule from a flower pollinated once and twice by the Dunnett's test $(P<0.05)$.

canadensis, the level of inbreeding depression was low (Culley, 2000). On the other hand, only the nototribic pollination (Beattie, 1974) was observed in my experiment. Beattie (1971) reports high pollen load on ventral parts of pollinators. Pollen grains on ventral parts adhere to stigmatic mucilage with a high possibility if pollinated sternotribically. Beattie (1969b) observed pollen grains in the stigmatic cavity of emasculated flowers. Cross-pollination may increase depending on the pollinator's activity. Flowers under open pollination formed a greater number of seeds than those hand-pollinated. Viola flowers under open pollination possibly produced the greater number of seeds in a capsule by repeated pollinations than flowers hand-pollinated that were pollinated once and the petals of which were removed. Viola set seeds slightly under a mosquito net. I observed that thrips that occasionally emerged

${ }^{\mathrm{x}}$ All petals were removed.
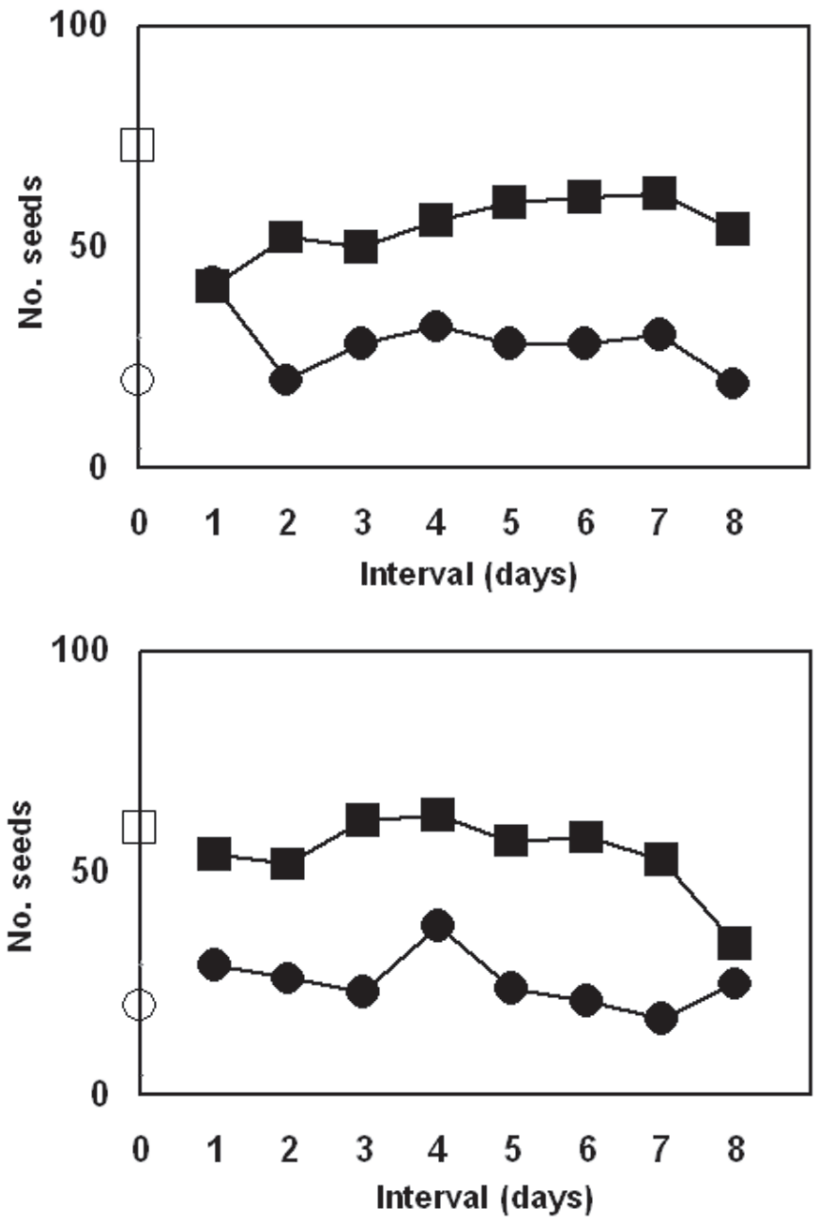

moved around anthers and stigmas.

Thus, viola flowers had seed producing ability throughout the long flowering period. Repeated pollinations seemed to be effective to increase the number of seeds in a capsule. In commercial seed production of ornamental plants, hand-pollination is often effective, but not practical for viola because of the large number of flowers on a plant and the small structure of each flower. Actually, pollinators are indispensable for commercial seed production of viola. For commercial seed production of viola, techniques of managing solitary bees that prefer viola are necessary. On the other hand, flowers under open pollination produced more seeds per capsule than flowers hand-pol-

Fig. 7. Maximum number of seeds formed by a flower pollinated with 30 pollen grains at full opening of the flower and pollinated again with 30 or a mass of pollen grains in two cultivars of viola. (upper) VCS, (lower) PB. The representation of shapes in graphs is the same with Fig. 4.

linated, but the number of seeds in a capsule was smaller than the number of ovules in a flower, which indicates that a considerable percentage of ovules abort. It is necessary to increase the number of seeds in a capsule by preventing ovule abortion to increase seed yield with less production cost by studying the possible causes of ovule abortion; which may include pollen limitation or resource limitation in viola.

Table 2. Seed production in two cultivars of viola subjected to six treatments.

\begin{tabular}{lcc}
\hline Treatment & Fruit set (\%) & No. mature seeds/flower \\
\hline Velour Cream Splash & & \\
Open pollination & $73.3 \mathrm{~b}^{\mathrm{z}}$ & $34.2 \mathrm{~b}^{\mathrm{y}}$ \\
Mosquito net cover & $0 \mathrm{c}$ & -- \\
Emasculation & $0 \mathrm{c}$ & -- \\
Hand-pollination $^{\text {Petal removal }}$ & $97.4 \mathrm{a}$ & $28.9 \mathrm{c}$ \\
Princess Blue & $0 \mathrm{c}$ & --- \\
Open pollination & & $45.9 \mathrm{a}$ \\
Mosquito net cover & $96.0 \mathrm{a}$ & $0.4 \mathrm{~d}$ \\
Emasculation & $12.5 \mathrm{c}$ & --- \\
Hand-pollination & $0 \mathrm{c}$ & $29.0 \mathrm{c}$ \\
Petal removal & $100 \mathrm{a}$ & --- \\
\hline
\end{tabular}

${ }^{2}$ Mean separation by Tukey's studentized range test using arcsin-transformed data $(P<0.05)$.

${ }^{y}$ Mean separation by Scheffe's test $(P<0.05)$. 

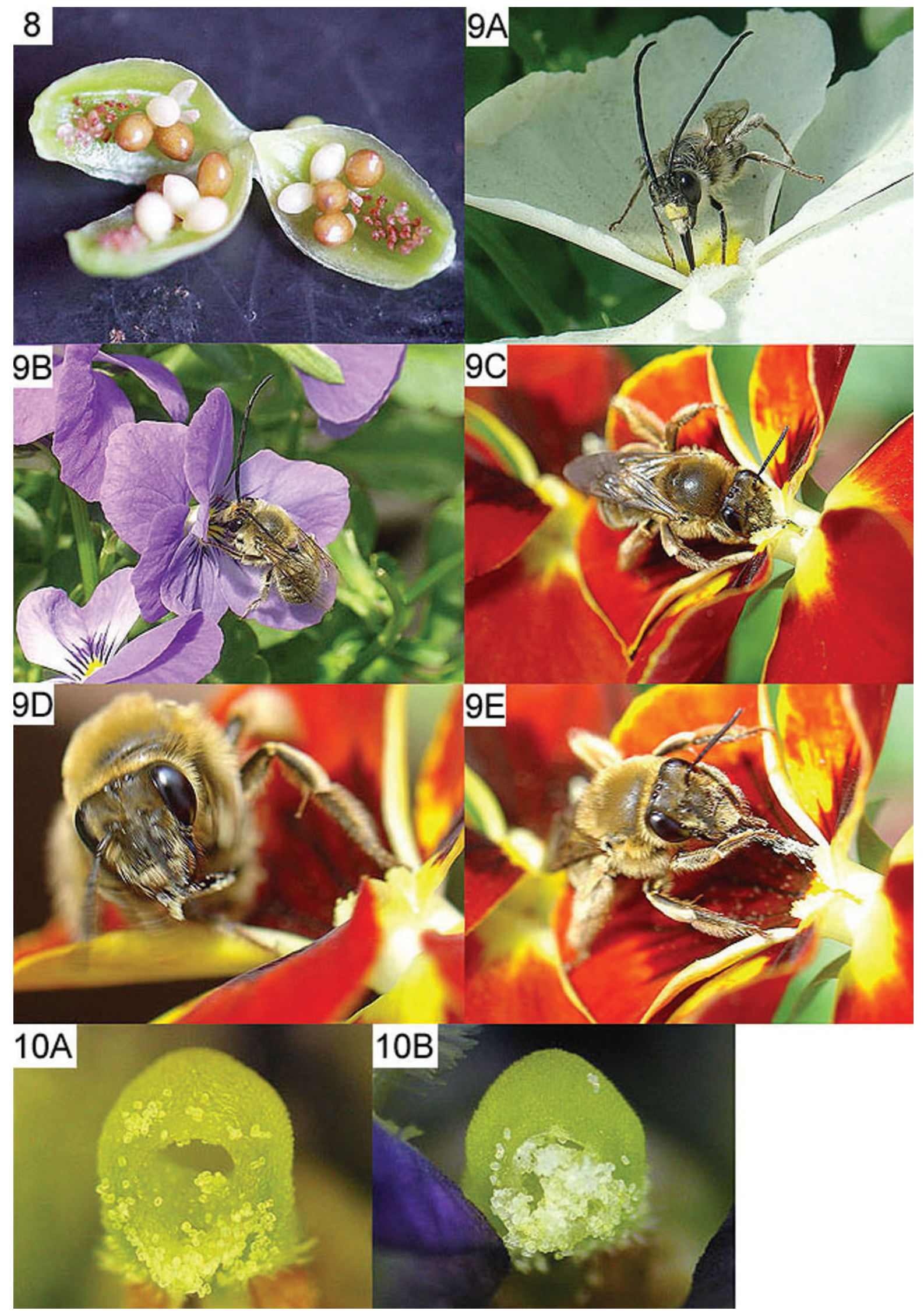

Figs. 8-10. (8) Seed development in the capsule pollinated twice and sampled at $20 \mathrm{~d}$ after the second pollination. The flower was pollinated with 30 pollen grains at full opening of the flower and was pollinated again with 30 pollen grains $8 \mathrm{~d}$ after the first pollination. There are three groups of ovules: brown developing seeds that were fertilized at the first pollination, white developing seeds that were fertilized at the second pollination, and aborted ovules. (9) Pollinators of viola observed in the experiment at field. (9A) Eucera spurcatipes. (9B) A Tetralonia nipponensis. (9C) Andrena nawai. Note that the head is contacting the entrance of the stigmatic cavity. (9D) Andrena nawai just after sucking the nectar. Note the pollen grains around the mouthparts. (9E) Andrena nawai wiping pollen grains on the proboscis. (10A and B) The stigma of viola after visitation by solitary bees. 


\section{Literature Cited}

Beattie,A.J. 1969a. The floral biology of three species of Viola. New Phytol. 68:1187-120.

Beattie, A.J. 1969b. Studies in the pollination ecology of Viola. I. The pollen-content of stigmatic cavities. Watsonia 7:142-156

Beattie, A.J. 1971. Pollination mechanisms in Viola. New Phytol. 70:343-360

Beattie, A.J. 1972a. Insect-visitors to three species of violet (Viola) in England. Entomol. Mon. Mag. 108:7-11.

Beattie, A.J. 1972b. The pollination ecology of Viola. II. Pollen loads of insect-visitors. Watsonia 9:13-25.

Beattie, A.J. 1974. Floral evolution in Viola. Ann. Mo. Bot. Gard. 61:781-793.

Beattie, A.J. 1976. Plant dispersion, pollination and gene flow in Viola. Oecologia 25:291-300.

Clark, D. G., C. Richards, Z. Hilioti, and K. Brown. 1997. Effect of pollination on accumulation of ACC synthase and ACC oxidase transcripts, ethylene production and flower petal abscission in geranium (Pelargonium $\times$ hortorum L.H Bailey). Plant Mol. Biol. 34:855-865.
Cruden, R.W. 1977. Pollen ovule ratios: Aconservative indicator of breeding systems in flowering plants. Evolution 31:32-46.

Culley, T.M. 2000. Inbreeding depression and floral type fitness differences in Viola canadensis (Violaceae), a species with chasmogamous and cleistogamous flowers Can. J. Bot. 78:1-10.

Freitas, L. and M. Sazima. 2003. Floral biology and pollination mechanisms in two viola species-From nectar to pollen flowers? Ann. Bot. 91:311--317.

Halevy, A.H. 1995. The role of sensitivity to ethylene in pollination-induced corolla senescence syndrome. Acta Hort. 405:210-215.

Hilioti, Z., C. Richards, and K.M. Brown. 2000. Regulation and role of pollination-induced ethylene on petal abscission of Pelargonium $\times$ hortorum. Physiol. Plant. 109:322-332.

Hoekstra, F.A. and G. Ch. van Roekel. 1986. Effects of interspecific pollination on stylar ethylene production and flower longevity in petunia hybrida. Acta Hort. 181:105-112.

Ishii, H.S. and S. Sakai. 2000. Optimal timing of flower abortion: Experimental study on Erythronium japoncum (Liliaceae). Func. Ecol.
14:122-128.

Ketsa, S. and A. Rugkong. 2000. Ethylene production, senescence and ethylene sensitivity of Dendrobium 'Pompadour'flowers following pollination. J. Hort. Sci. Biotechnol. 75:149-153.

Larsen P.B., E.N. Ashworth, M.L. Jones, and W.R. Woodson. 1995. Pollination-induced ethylene in carnation: Role of pollen tube growth and sexual compatibility. Plant Physiol. 108:1405-1412.

Piskornik, Z. 1986. The role of ethylene in the pollination and senescence of flowers of bulbous plants. Acta Hort. 181:407-414.

van Doorn W.G. and A.D. Stead. 1997. Abscission of flowers and floral parts. J. Expt. Bot. 48:821-827

van Doorn W.G. 2002. Effect of ethylene on flower abscission: a survey. Ann. Bot. 89:689-693.

Vivian-Smith A., M. Luo, A. Chaudhury, and A. Koltunow. 2001. Fruit development is actively restricted in the absence of fertilization in Arabidopsis. Development 128:2321-2331.

Woodson, W.R., K.Y. Park, A. Drory, P.B. Larsen, and H. Wang. 1992. Expression of ethylene biosynthetic pathway transcripts in senescing carnation flowers. Plant Physiol. 99:526-532. 